\title{
Assessing the Genetic Diversity of Chrysanthemum Cultivars with Microsatellites
}

\author{
Yuan Zhang ${ }^{1}$ and Chen Wang ${ }^{1}$ \\ College of Landscape Architecture, Beijing Forestry University, No. 35 Tsinghua East Road, Haidian \\ District, Beijing 100083, China \\ HongZheng Ma \\ Institute of Botany, the Chinese Academy of Sciences, Beijing 100093, China \\ SiLan Dai ${ }^{2}$ \\ College of Landscape Architecture, Beijing Forestry University, No. 35 Tsinghua East Road, Haidian \\ District, Beijing 100083, China
}

\begin{abstract}
AdDITIONAL INDEX WORDS. capillary electrophoresis, Chrysanthemum $\times$ morifolium, cultivar classification, genomic library, magnetic bead, simple sequence repeat

ABSTRaCt. The morphological characteristics of chrysanthemum (Chrysanthemum $\times$ morifolium) are rich in variation. However, as a result of the aneuploid polyploidy of the chrysanthemum genome and the lack of proper tools, the genomic information of this crop is limited. Development of microsatellite markers has been an increasing trend in crop genetic studies because of the applicability of these markers in breeding programs. In this study, we reported the development of a simple sequence repeat in chrysanthemums using a magnetic beads enrichment method. An enriched genomic library with AC and GT microsatellite motifs was constructed, and 53 positive clones were detected by a colony polymerase chain reaction (PCR) technique. Of these clones, 35 showed high-quality sequences, and 35 primer pairs were designed accordingly. Twenty-six $(\mathbf{7 4 . 2 9 \%})$ of the 35 primer pairs revealed polymorphisms on a set of 40 chrysanthemum cultivars. There were 172 alleles amplified over 26 loci with an average of 6.615 alleles per locus. The mean values of gene diversity corrected for the sample size and the inbreeding coefficient were 0.609 and 0.119 over 26 loci, respectively, which indicated that the majority of the microsatellite loci is highly informative. Cluster analysis based on 26 polymorphic loci demonstrated that the selected cultivars were clustered according to geographical origin. This study shows the isolation efficiency of the magnetic beads technique; the abundance of microsatellites in chrysanthemum; and the potential application for the cultivar classification, the studies on genetic diversity, and molecular breeding of chrysanthemums, which is beneficial to promoting the conservation and sustainable use of this crop.
\end{abstract}

Chrysanthemum is a famous traditional flower in China that possesses a high aesthetic value and abundant cultural associations (Zhang et al., 2013). Because of the active dissemination and breeding of this flower around the world, an ample germplasm resource for chrysanthemums has been built. Currently, the number of chrysanthemum cultivars worldwide is $\approx 20,000$ to 30,000 (Anderson, 2006). In China, chrysanthemums were traditionally classified into five petal types such as flat, spoon, tubular, anemone, and peculiar (Chinese Chrysanthemum Association, 1993). Recently, nine color classification groups were also introduced by Hong et al. (2012). Because of the characteristics of large showy flowers, rich flower colors, various cultivars with multiple variations, strong resistance, and abundant cultural connotation, chrysanthemums are becoming increasingly visible in many countries.

During the last 5 years, many studies on chrysanthemums have been conducted, mainly focused on the aspects of morphology (Banerji et al., 2012; Hong et al., 2012), cytology

Received for publication 7 Aug. 2013. Accepted for publication 11 Sept. 2013. This study was supported by the National Special Research Project for Forestry Public Affairs (Grant No. 200904050).

We are sincerely grateful to Beijing Dadongliu Nursery for providing plant materials.

${ }^{1}$ Yuan Zhang and Chen Wang contributed equally to this work.

${ }^{2}$ Corresponding author. E-mail: silandai@sina.com.
(El-Twab and Kondo, 2012; Zhang et al., 2013), biochemistry (Liang et al., 2010; Lin and Harnly, 2010), physiology (Leiss et al., 2009), and functional genomics (Chen et al., 2012; Gu et al., 2011). However, studies on the molecular genetics of chrysanthemums have rarely been reported because of the aneuploid polyploidy of the chrysanthemum genome, which indicates the non-exponential loss or gain of chromosomes that results from the hybridization and mutation of chrysanthemum cultivars. Therefore, the genomic information of this crop is very limited. Moreover, chrysanthemum cultivars have been evaluated with respect to many morphological traits and morphometric criteria, but very little work has been performed on the molecular characterization of the chrysanthemum genome. It is therefore necessary to develop and use tools to evaluate the genetic diversity among chrysanthemum cultivars to promote the conservation and sustainable use of this crop.

Currently, the application of codominant markers in chrysanthemums is very limited. Among dozens of molecular marker techniques, simple sequence repeat (SSR) markers have gained considerable popularity in plant genetics and breeding because of many desirable attributes, including hypervariability, a multiallelic nature, codominant inheritance, reproducibility, relative abundance, extensive genome coverage (including organellar genomes), chromosome specific location, amenability to automation, and high throughput genotyping (Kalia et al., 2011). However, the application of SSR markers on the 
chrysanthemum genome is rather difficult because most of the large-flowered chrysanthemum cultivars are hexaploid-based aneuploid (Zhang et al., 2013), indicating a complicated genetic background. Therefore, the read and data analysis is a major problem for the SSR analysis of chrysanthemums. Fortunately, a powerful method called microsatellite DNA allele countingpeak ratios (MAC-PR) was proposed by Esselink et al. (2004) to calculate the ratios between the peak areas for two alleles in all of the samples where these two alleles occurred together. The observed allele peak ratios are then plotted in a histogram, and the histograms that produce at least two wellseparated groups are selected for further analysis.

Another drawback to using microsatellites is that these sequences must be isolated de novo from species that are being examined for the first time, and such isolation can be timeconsuming. This drawback has recently been overcome by the introduction of library enrichment and automatic sequencing (Zane et al., 2002). In recent years, several strategies for microsatellite isolation have been developed. Screening genomic libraries by hybridization with microsatellite probes and sequencing the hybridized positive clones is a traditional but laborious and costly approach (Billotte et al., 2001). In contrast, screening microsatellite-enriched, small-insert libraries can significantly reduce the time and cost (Billotte et al., 2001). Enrichment can be performed by hybridizing microsatellite-containing fragments with biotin-labeled probes that are captured by magnetic beads coated with streptavidin or fixed on a nitrate filter (Edwards et al., 1996). The eluted portion that remains after removing the non-hybridized DNA is highly enriched for microsatellites (Butcher et al., 2000). Among the isolation methods, this enrichment method appears to be highly efficient (Zane et al., 2002) and has been successfully applied to many crop species such as Punica granatum (Ebrahimi et al., 2010), Avicennia germinans (Mori et al., 2010), Taihangia rupestris (Wang et al., 2010a), and Cathaya argyrophylla (Wang et al., 2010b).

The objectives of this study were 1) to isolate and identify microsatellites from the chrysanthemum genome; 2) to detect polymorphisms using the novel microsatellites in a collection of chrysanthemum cultivars with different petal types and flower colors through a superior analysis. Nursery (Beijing, China).

${ }^{x}$ Hong et al. (2012).

wZhang et al. (2013). method for the read and analysis of data; and 3) to reveal the genetic diversity among the selected cultivars.

\section{Materials and Methods}

Plant materials and DNA isolation. A total of 40 chrysanthemum cultivars were selected on the basis of two morphological traits (petal type and flower color), including 30 Chinese traditional cultivars and 10 Japanese cultivars (Table 1).

Table 1. The name, petal type, flower color, and ploidy level of selected Chrysanthemum $\times$ morifolium cultivars used for the applicability test of simple sequence repeat markers and the genetic diversity

\begin{tabular}{|c|c|c|c|c|}
\hline Sample code & Cultivar & Petal type $^{y}$ & Flower color $^{x}$ & Ploidy level $^{\mathrm{w}}$ \\
\hline $\mathrm{C} 22$ & Wenyuanyinghua & Tubular & Pink & - \\
\hline $\mathrm{C} 50$ & Tianewu & Flat & Yellow & - \\
\hline C69 & Baiouzhubo & Spoon & White & - \\
\hline $\mathrm{C} 77$ & Taoliuchunyi & Spoon & Dark red & - \\
\hline $\mathrm{C} 88$ & Yuniangsuiyi & Tubular & Dark red & Hexaploid-based aneuploid \\
\hline $\mathrm{C} 95$ & Jinxianchuizhu & Tubular & Yellow & - \\
\hline $\mathrm{C} 117$ & Jinmudan & Spoon & Yellow & - \\
\hline $\mathrm{C} 137$ & Furongtuogui & Spoon & Pink & Hexaploid-based aneuploid \\
\hline $\mathrm{C} 210$ & Midiansongqi & Tubular & Yellow & - \\
\hline $\mathrm{C} 224$ & Yuezhonggui & Anemone & Yellow & - \\
\hline $\mathrm{C} 241$ & Damofeiying & Tubular & Dark red & Hexaploid-based aneuploid \\
\hline $\mathrm{C} 272$ & Zhuzijiaolong & Peculiar & Dark red & Hexaploid-based aneuploid \\
\hline $\mathrm{C} 278$ & Queshetuogui & Anemone & Pink & Hexaploid-based aneuploid \\
\hline C288 & Dujuanjixue & Flat & White & - \\
\hline C298 & Huangchangfeng & Flat & Yellow & - \\
\hline C300 & Lijin & Flat & Red & Pentaploid-based aneuploid \\
\hline C316 & Hongyijinxiu & Tubular & Dark red & - \\
\hline C344 & Deyiyuan & Spoon & Pink & Hexaploid-based aneuploid \\
\hline C353 & Moxie & Tubular & Dark red & Hexaploid-based aneuploid \\
\hline C364 & Yinxuetai & Flat & White & - \\
\hline C372 & Jingetiema & Peculiar & Yellow & - \\
\hline C389 & Baiyuzhulian & Tubular & White & - \\
\hline C404 & Tangyuqiushi & Flat & White & - \\
\hline $\mathrm{C} 441$ & Dahongmaoju & Spoon & Red & - \\
\hline $\mathrm{C} 442$ & Honglianranjin & Flat & Red & - \\
\hline $\mathrm{C} 459$ & Jindingtiaoyuan & Flat & Red & - \\
\hline C500 & Biyutuogui & Anemone & Green & - \\
\hline $\mathrm{C} 748$ & Tangyujinhua & Flat & Yellow & - \\
\hline $\mathrm{C} 801$ & Fenyun & Flat & Pink & - \\
\hline $\mathrm{C} 803$ & Cuiwu & Tubular & Green & - \\
\hline J013 & Taipingjinri & Flat & Yellow & - \\
\hline J017 & Jiumidemeng & Spoon & Pink & - \\
\hline J019 & Guohuawanlei & Flat & White & - \\
\hline $\mathrm{J} 023$ & Guohuazhuchuan & Spoon & Yellow & - \\
\hline J034 & Caoangudian & Flat & Yellow & - \\
\hline J042 & Jingxingyoujin & Flat & Yellow & Hexaploid-based aneuploid \\
\hline J049 & Donghaifuzhou & Flat & White & - \\
\hline J052 & Guohuachuying & Flat & Pink & - \\
\hline J072 & Guohuayingxiong & Flat & White & - \\
\hline $\mathrm{J} 101$ & Taipingzhenshi & Flat & White & - \\
\hline
\end{tabular}


with a "J" represent Japanese cultivars. All samples were collected from the Beijing Dadongliu

'Ministry of Agriculture of the People's Republic of China (2002).

"_." represents unknown ploidy level. 
The classification criteria for petal type and flower color were based on the Ministry of Agriculture of the People's Republic of China (2002) and Hong et al. (2012), respectively, and the ploidy level of some known cultivars was based on Zhang et al. (2013). Fresh, young leaf samples from each of these cultivars were independently collected from our chrysanthemum conservation nursery in Beijing. The total genomic DNA of each cultivar was extracted from the fresh leaves using the Extractive Kit for Plant Genomic DNA (Tiangen Biotech Co., Beijing, China) with minor modifications, and the DNA of chrysanthemum cultivar Jinbeidahong was used to develop the microsatellite-enriched libraries.

ENRICHED LIBRARY CONSTRUCTION. Microsatellites were isolated following the protocol of Hamilton et al. (1999) with some important modifications. Briefly, $20 \mu \mathrm{g}$ of genomic DNA was digested in a mix of four restriction enzymes (NheI, AluI, RsaI, and HaeIII) that yielded fragments between 200 and $1000 \mathrm{bp}$ in length. The single-stranded overhangs of the obtained DNA fragments were removed using mung bean nuclease, and the genomic DNA ends were then dephosphorylated by calf intestinal alkaline phosphatase $(0.01 \mathrm{U} / \mathrm{pmol} \mathrm{DNA})$ and ligated to phosphorylated SNX linkers to provide a PCR priming site. $X m n I$ was also included in the ligation procedure. The linkerligated fragments were amplified by PCR using SNX-forward (5'CTAAGGCCTTGCTAGCAGAAGC3') as the primer. These fragments were heat-denatured and then hydrolyzed with biotinylated $(\mathrm{AC})_{15}$ at $65^{\circ} \mathrm{C}$ and $(\mathrm{GT})_{10}$ at $62{ }^{\circ} \mathrm{C}$ for $\approx 17 \mathrm{~h}$ in a hybridization oven. Then, $15 \mu \mathrm{g}$ of streptavidin-coated magnetic beads was washed three times with $300 \mu \mathrm{L}$ of $0.5 \times$ saline sodium citrate (SSC) for $5 \mathrm{~min}$ per wash and suspended in $100 \mu \mathrm{L}$ of $0.5 \mathrm{X}$ SSC. The hybridization solution was added and incubated on a heat block at $43^{\circ} \mathrm{C}$ for $1 \mathrm{~h}$ and then agitated for $5 \mathrm{~min}$. The solution was then placed in an oven at a suitable temperature ( $68^{\circ} \mathrm{C}$ for $\mathrm{AC}$ and $62^{\circ} \mathrm{C}$ for GT motifs) for hybridization. The solution was washed twice with $200 \mu \mathrm{L}$ of $2 \times \mathrm{SSC}$, twice with $200 \mu \mathrm{L}$ of $1 \times \mathrm{SSC}$, and four times with $200 \mu \mathrm{L}$ of $0.5 \times \mathrm{SSC}$ for 5 min per wash to remove non-targeted DNA from the oligobead complex. The beads were separated from the solution between washes using a MagneSphere Magnetic Separation Stand (Jinbolai Biotech Co., Beijing, China). Microsatelliteenriched DNA was eluted by adding $30 \mu \mathrm{L}$ of preheated sterile water at $98{ }^{\circ} \mathrm{C}$, incubating at $98{ }^{\circ} \mathrm{C}$ for $15 \mathrm{~min}$, and then separating the solute from the beads. The recovered single-stranded DNA was amplified using the SNX forward linker as a primer. The resulting double-stranded DNA products were digested with NheI to produce cloning ends. The repeat-enriched DNA was ligated into an XbaI-digested dephosphorylated pBluescript SK (+/-) vector, transformed into competent Escherichia coli (MC1061) cells, and plated on LB medium containing $10 \mathrm{mg} \cdot \mathrm{mL}^{-1}$ ampicillin, $20 \mathrm{mg} \cdot \mathrm{mL}^{-1} \mathrm{X}$-gal, and $23 \mathrm{mg} \cdot \mathrm{mL}^{-1}$ isopropyl $\beta$-Dthiogalactoside. After $17 \mathrm{~h}$, the transformed microsatellite clones (white clones) were set on new plates.

Sequencing AND PRimer Design. Colony PCR was used to identify the positive clones (Sheu et al., 2000). The clones with 500 to $800 \mathrm{bp}$ inserts were selected and sequenced (Ruiboxingke Biotech Co., Beijing, China), and the sequences were analyzed using Chromas software (Version 2.4.1; Technelysium, South Brisbane, Australia). Primers were designed for sequences that contained microsatellite repeats using Primer software (Version 5.0; Premier Biosoft, Palo Alto, CA). The designed primers were synthesized commercially by Sangon Biotech Co. (Shanghai, China).
PCR AMPLIFICATION AND ELECTROPHORESIS. The efficiency of designed primers was assessed among eight chrysanthemum cultivars containing four Chinese traditional cultivars and four Japanese cultivars. The PCR reaction system was based on $\mathrm{Li}$ et al. (2012) with some important modifications. Briefly, $100 \mathrm{ng}$ DNA template, $2.0 \mathrm{mmol} \cdot \mathrm{L}^{-1} \mathrm{Mg}^{2+}, 0.1 \mathrm{mmol} \cdot \mathrm{L}^{-1} \mathrm{dNTP}$, $0.5 \mu \mathrm{mol} \cdot \mathrm{L}^{-1}$ each of forward and reverse primers, and $1 \mathrm{U} \mathrm{Taq}$ DNA polymerase were mixed in a $20 \mu \mathrm{L}$ reaction system. All reactions were performed using a TProfessional Standard Gradient Thermocycler (Biometra, Goettingen, Germany). The following thermal cycling protocol was used: initial melting at $94{ }^{\circ} \mathrm{C}$ for $5 \mathrm{~min}$, followed by 35 cycles at $94{ }^{\circ} \mathrm{C}$ for $50 \mathrm{~s}$, and then $45 \mathrm{~s}$ at the optimum annealing temperature, which was determined by testing in the range of $\pm 5^{\circ} \mathrm{C}$ from the theoretical annealing temperature and then $72^{\circ} \mathrm{C}$ for $50 \mathrm{~s}$. Then, the reaction mixture was held at $4{ }^{\circ} \mathrm{C}$ after a final extension step of $72{ }^{\circ} \mathrm{C}$ for $10 \mathrm{~min}$. Amplified PCR products were separated using agarose gel electrophoresis (Ruiboxingke Biotech Co.).

Data ANAlysis and Genetic Diversity anALysis. The 40 chrysanthemum cultivars mentioned were used to test the practicability and polymorphisms of the designed primers by capillary electrophoresis (CE) to conversely reveal the genetic diversity among the cultivars. The original data, with an $f_{s} a$ format, were obtained after the analysis of the amplification products by GeneMarker software (Version 1.65; SoftGenetics, State College, PA); then the interpretative data, with an $x l s$ format, were obtained by using the MAC-PR method (Esselink et al., 2004). The number of valid alleles (NA) with the gene diversity corrected for sample size (HE; Nei, 1978) and inbreeding coefficient [IC (Leutenegger et al., 2003)] of the polymorphic loci were analyzed using Polysat [Version 1.3 (Clark and Jasieniuk, 2011)] and Excel 2010 (Microsoft, Redmond, WA) software. Finally, R software (Version 3.0.1; Parametric Technology, Needham, MA) was used to construct two neighbor joining (NJ) trees for 40 cultivars by Lynch distance and Bruvo distance.

\section{Results}

The CONSTRUCTION OF AN ENRICHED GENOMIC LIBRARY. An enriched genomic library with dinucleotide AC and GT microsatellite motifs was constructed. Among the observed clones, 53 positive clones were selected using colony PCR and were sequenced. The sequences of 18 clones were insufficiently clear, whereas the SSR motifs of other 35 clones with high-quality sequences were observed. All of the 35 sequenced clones were suitable for primer design, which showed five types of motifs: CA, TC, AG, GT, and GAT with the maximum replication times of $21,10,30,10$, and four, respectively (Table 2).

To uncover the optimum annealing temperature of each primer pair, eight chrysanthemum cultivars (four Chinese traditional cultivars and four Japanese cultivars) were tested in the range of $\pm 5{ }^{\circ} \mathrm{C}$ from the theoretical annealing temperature of each primer pair. According to the screening results, the optimum annealing temperatures of these primer pairs ranged from 46 to $58{ }^{\circ} \mathrm{C}$ (Table 2 ).

THE POLYMORPHISM TEST OF DESIGNED PRIMERS. As a result of the low resolution ratio of agarose gel, CE was adopted to test the polymorphism of designed primers among the 40 chrysanthemum cultivars. The method for the read and analysis of the data was based on Esselink et al. (2004). Nine (25.71\%) primer 
Table 2. Summary of the motif repeat, range of product length, optimum annealing temp (Tm), number of alleles (NA), gene diversity coefficient (HE), inbreeding coefficient (IC), and primer sequences of the 26 polymorphic primer pairs.



pairs were monomorphic, and $26(74.29 \%)$ primer pairs showed a polymorphism pattern in selected cultivars (Fig. 1).

The DIVERSITY OF THE POLYMORPHIC PRIMERs. The diversity of the 26 polymorphic primer pairs was assessed among the
40 chrysanthemum cultivars. According to the results, a total of 172 alleles were mapped to 26 loci. The NA, HE, and IC at 26 polymorphic microsatellite loci observed among the 40 chrysanthemum cultivars is given in Table 2 . 




respectively; whereas 1, 2, and $\mathbf{3}$ represent chrysanthemum cultivars coded J019, C288, and C353, respectively. The x-axis and y-axis are the size of alleles and intensity of fluorescence, respectively.

The observed number of valid alleles per locus ranged from two to 16 with an average of 6.615 alleles per locus, the observed $\mathrm{HE}$ values ranged from 0.052 to 0.885 with an average of 0.609 , and the IC values were estimated to range from -0.196 to 0.699 with an average of 0.119 . The DF value of NA was the highest (3.699) compared with the values of HE (0.241) and IC (0.266).

The genetic diversity aMong 40 Chrysanthemum CULTIVARS BASED ON THE GENETIC DISTANCES OF ALL POLYMORPHIC LOCI. The genetic diversity among the 40 chrysanthemum cultivars was analyzed by two NJ trees (Lynch distance and Bruvo distance) based on the genetic distances of 26 polymorphic loci (Fig. 2). Both methods classified the 40 selected cultivars into two branches.

For both methods, cultivars with different petal types and flower colors clustered dispersedly into two branches. Therefore, it seems that these loci were not correlated with the petal type and flower color of the selected cultivars. However, all of the Japanese cultivars clustered together in branch I, indicating that the cultivars clustered according to the geographical origin. Moreover, all of the cultivars belonging to the anemone group and most of the dark red cultivars $(85.33 \%)$, which are the Chinaspecific chrysanthemum germplasm, closely clustered in branch II, further confirming the abundant diversity of chrysanthemums originating from different geographical regions.

\section{Discussion}

Molecular markers for Chrysanthemum. Efforts have been made to identify chrysanthemum cultivars, estimate the genetic distances, and discuss the origin of modern cultivars using DNA markers, mainly using random amplified polymorphic DNA (RAPD), amplified fragment length polymorphism (AFLP), intersimple sequence repeat (ISSR), and sequencerelated amplified polymorphism (SRAP) markers.
Huang et al. (2000) used 45 random primers to detect molecular markers in three hybrid combinations of chrysanthemums by using RAPD markers. This study found that the patterns of molecular markers could be classified into seven types, and $34.4 \%$ to $48.9 \%$ of the RAPD markers were found to reveal additivity among parents and offspring in all samples. However, $38 \%$ to $52.6 \%$ of the markers were absent in the offspring, but $11.6 \%$ to $13.1 \%$ of the unique markers were present in the offspring. Martí et al. (2002) found that 15 commercial chrysanthemum cultivars could be distinguished from each other through RAPD analysis, and the level of similarity between the cultivars was low. Sehrawat et al. (2003) classified 13 chrysanthemum cultivars into two primary groups using RAPD markers and found that the results were consistent with the morphological differences and geographical distributions. In another study, the molecular characterizations of 10 new radiomutants of chrysanthemum were analyzed (LemaRumiñska et al., 2004). This study found that the original cultivar Richmond differed in genetic distance from the Lady Group mutants, and the analysis of genetic similarity indices revealed low diversity within the radiomutants. Moreover, the dendrogram obtained after cluster analysis separated the new cultivars as a group that differed from the original Richmond cultivar. The Lady Group cultivars, derived from the original cultivar by radiomutation, could be distinguished from each other by using RAPD markers of only a single primer or sets of two or three primers.

Kang et al. (2013) compared the polymorphisms, genetic diversity, and genetic distances between the original chrysanthemum cultivar and gamma-irradiated in vitro plants using AFLP markers and identified $83 \%$ (866 bands) of the polymorphisms using 12 primer combinations.

ISSR showed sufficient polymorphism to distinguish between various cultivars of chrysanthemums (Wolff et al., 1995). The diversity and genetic diversity among 29 populations of 



Fig. 2. The genetic diversity among the 40 selected Chrysanthemum ×morifolium cultivars shown in neighbor joining tree based on the genetic distances of all polymorphic loci: (A) Lynch distance, (B) Bruvo distance. Five groups of petal types are shown with different label shapes: flat, spoon, tubular, anemone, and peculiar group are shown with squares, equilateral triangles, circles, inverted triangles, and rhombuses, respectively. The color of the labels indicates different groups of flower color, and the white and dark red groups are shown as hollow and black, respectively.

chrysanthemum, one of Chrysanthemum indicum, and one of Chrysanthemum nankingense from China were analyzed using morphological traits and molecular markers. Twenty morphological traits and 182 ISSR marker fragments were scored, as amplified by 22 primers, and 243 SRAP marker fragments were generated by 26 primer pairs. Cluster analysis showed that the groupings of the accessions according to all four methods correlated well with the geographic region of origin, and most populations from the south of China were classified into one cluster, whereas most populations from the north of China were classified into another cluster (Shao et al., 2010).

SRAP markers were another powerful tool that were used for quantitative trait locus (QTL) analysis on chrysanthemums. A segregating population of $142 \mathrm{~F}_{1}$ progeny of the cross between two chrysanthemum cultivars, Yuhualuoying and Aoyunhanxiao, was used to construct two separate genetic linkage maps through a double pseudo-test cross mapping strategy, and the genotyping was performed using 500 SRAP primer combinations, of which $\approx 50 \%$ were informative (Zhang et al., 2011).

From these studies, we can see that RAPD, AFLP, ISSR, and SRAP markers are useful tools for the molecular research on chrysanthemums. The polymorphic loci ranged from $69 \%$ to $99.6 \%$, which indicates that the variations in the genomic DNA are spread throughout the evolutionary progress of the chrysanthemum and that an ample genetic diversity does exist in the chrysanthemum germplasm. Although these markers have been successfully used for cultivar identification, the sample sizes of these studies were deficient and could not cover the entire genetic background of the chrysanthemum. Moreover, because the performances of morphological characters are the result of the expression of certain functional genes under the internal and external environment, the structural differences of the DNA are not always exposed. The SSR marker technique could resolve these problems to a large extent.

MAGNETIC BEAdS ENRICHMENT METHOD FOR DEVELOPING SPECIFIC PRIMERS FOR CHRYSANTHEMUM. Generally, SSR is classified into two types: genomic SSR (gSSR) and expressed sequence tags SSR (EST-SSR). Compared with gSSR, ESTSSR possesses stronger transferability among genera and species (Lindqvist et al., 2006). The development of EST-SSR has been successfully applied to many species such as Hevea brasiliensis (Feng et al., 2009), Humulus lupulus (Koelling et al., 2012), and C. nankingense (Wang et al., 2013). However, compared with gSSR, most of the repeat sequences of EST-SSR are shorter, and the polymorphism and specificity of the microsatellites are much poorer (Eujayl et al., 2001), which is not suitable for the chrysanthemums that possess complicated genetic backgrounds. In the present study, we sought to increase the efficiency of gSSR isolation from the chrysanthemum genome by using SSR enrichment. In doing so, we evaluated the effectiveness of an SSR-enriched library. By testing various combinations of 4-bp and 6-bp restriction enzymes, the four restriction enzymes that yielded DNA fragments between 200 and $1000 \mathrm{bp}$ in length (NheI, AluI, RsaI, and HaeIII) were selected as beneficial fragments for enrichment and cloning.

There was a low percentage of clones lacking microsatellite repeats $(34 \%)$ in comparison with the average percentage observed in enriched libraries (36\%) (Squirrell et al., 2003), indicating that the efficiency of the enrichment step was higher in this study and that the screening of the clones before sequencing was effective. Therefore, the colony PCR technique seems to be a suitable method for screening clones before sequencing. 
The magnetic bead enrichment method is a more efficient technique than other enrichment methods for identifying microsatellite motifs (Zane et al., 2002). In the present study, the mean number of NA, HE, and IC suggests a high degree of variation in the isolated loci. These markers can thus be classified as informative.

The electrophoresis Method AND MAC-PR METHOD. Agarose gel, sodium dodecyl sulfate polyacrylamide gel, and capillary are three basic media for electrophoresis in molecular marker research (Grossman and Colburn, 1992; Johansson, 1972; Shapiro et al., 1967). Agarose gel electrophoresis is simple and time-efficient, but the resolution ratio of this method is much lower in cooperation with the other methods. As a result of the high resolution ratio and repeatability, sodium dodecyl sulfate polyacrylamide gel electrophoresis (SDS-PAGE) is being widely used in molecular marker research for many different crops. However, SDS-PAGE cannot reveal much genetic information about the complicated genetic background of chrysanthemums. CE offers high efficiency, high speed, microscale, multiple models, cost-effectiveness, and automation, which is suitable for the molecular research on polyploid species. In the present study, we analyzed the genetic diversity of 40 chrysanthemum cultivars based on the genetic distances of all of the polymorphic loci by $\mathrm{CE}$ and obtained commendable results.

The ploidy of the large-flowered chrysanthemum cultivars ranged from pentaploid to octoploid, but most of the cultivars exhibited hexaploid-based aneuploidy (Zhang et al., 2013). Therefore, different from the species with simple genetic background (e.g., diploidy species), the read and analysis of the data for chrysanthemums by SSR marker technique are confusing. MAC-PR (Esselink et al., 2004) was approved as a powerful method for SSR analysis of the chrysanthemum in our study. According to the CE results (Fig. 1), one, two, three, or six peaks could appear for monomorphic loci, which are the common divisors of natural number six. If a certain cultivar is aneuploid, we could read the peak values to justify its polymorphism. Using this approach, we were able to assign precise allelic configurations (the actual genotype) to almost all of the cultivars analyzed for the multiple loci investigated. Moreover, MAC-PR also appears to be a very effective tool for detecting null alleles in polyploidy species (Esselink et al., 2004).

The Genetic Diversity AMONG 40 Chrysanthemum CULTIVARs. Roxas et al. (1993) analyzed the relationship between the petal type and the isozyme zymogram of higo chrysanthemums ( $C$. grandiflora) using SDS-PAGE and determined that there was no direct relativity between the petal type and the isozyme zymogram, which was consistent with our results. Ebrahimi et al. (2010) also found that in most cases, the cluster results of 13 polymorphic loci were not correlated with the morphological traits of 13 pomegranate $(P$. granatum) cultivars. In the present study, all of the Japanese cultivars were clustered together in branch I; moreover, the Chinese-specific chrysanthemum germplasm, anemone, and dark red cultivars, closely clustered in branch II, indicating that the cultivars clustered according to the geographical origin, which is consistent with the results of Sehrawat et al. (2003) and Shao et al. (2010).

\section{Literature Cited}

Abd El-Twab, M.H. and K. Kondo. 2012. Physical mapping of 5S and 45S rDNA in Chrysanthemum and related genera of the Anthemideae by FISH, and species relationships. J. Genet. 91:245-249.
Anderson, N.O. 2006. Chrysanthemum (Chrysanthemum $\times$ grandiflora Tzvelv.), p. 389-437. In: Anderson, N.O. (ed.). Flower breeding and genetics issues, challenges and opportunities for the 21 st century. Springer-Verlag, New York, NY.

Banerji, B.K., A. Batra, and A.K. Dwivedi. 2012. Morphological and biochemical characterization of chrysanthemum. J. Hort. Sci. 7:51-55.

Billotte, N., A.M. Risterucci, E. Barcelos, J.L. Noyer, P. Amblard, and F.C. Baurens. 2001. Development, characterization, and across-taxa utility of oil palm (Elaeis guineensis Jacq.) microsatellite markers. Genome 44:413-425.

Butcher, P.A., S. Decroocq, Y. Gray, and G.F. Moran. 2000. Development, inheritance and cross-species amplification of microsatellite markers from Acacia mangium. Theor. Appl. Genet. 101:1282-1290.

Chen, S., C. Li, X. Zhu, Y. Deng, W. Sun, L. Wang, F. Chen, and Z. Zhang. 2012. The identification of flavonoids and the expression of genes of anthocyanin biosynthesis in the chrysanthemum flowers. Biol. Plant. 56:458-464.

Chinese Chrysanthemum Association. 1993. Classification of chrysanthemum in China. Chinese Chrysanthemum Assn., Beijing Bureau Parks Woods, Beijing, China.

Clark, L.V. and M. Jasieniuk. 2011. POLYSAT: An R package for polyploid microsatellite analysis. Mol. Ecol. Resources 11:562-566.

Ebrahimi, S., B.E. Sayed-Tabatabaei, and B. Sharifnabi. 2010. Microsatellite isolation and characterization in pomegranate (Punica granatum L.). Iranian J. Biotechnol. 8:156-163.

Edwards, K.J., J.H. Barker, A. Daly, C. Jones, and A. Karp. 1996. Microsatellite libraries enriched for several microsatellite sequences in plants. Biotechniques 20:758-760.

Esselink, G.D., H. Nybom, and B. Vosman. 2004. Assignment of allelic configuration in polyploids using the MAC-PR (microsatellite DNA allele counting — peak ratios) method. Theor. Appl. Genet. 109:402-408.

Eujayl, I., M.E. Sorrells, M. Baum, P. Wolters, and W. Powell. 2001. Assessment of genotypic variation among cultivated durum wheat based on EST-SSRs and genomic SSRs. Euphytica 119:39-43.

Feng, S., W. Li, H. Huang, J. Wang, and Y. Wu. 2009. Development, characterization and cross-species/genera transferability of EST-SSR markers for rubber tree (Hevea brasiliensis). Mol. Breed. 23:85-97.

Grossman, P.D. and J.C. Colburn. 1992. Capillary electrophoresis: Theory and practice. Elsevier, Amsterdam, The Netherlands.

Gu, C., S. Chen, Z. Liu, H. Shan, H. Luo, Z. Guan, and F. Chen. 2011. Reference gene selection for quantitative real-time PCR in chrysanthemum subjected to biotic and abiotic stress. Mol. Biotechnol. 49: 192-197.

Hamilton, M.B., E.L. Pincus, A. Di Fiore, and R.C. Fleischer. 1999. Universal linker and ligation procedures for construction of genomic DNA libraries enriched for microsatellites. Biotechniques 27:500.

Hong, Y., X. Bai, W. Sun, F. Jia, and S. Dai. 2012. The numerical classification of chrysanthemum flower color phenotype. Hort. Sinica 39:1330-1340 [abstr.].

Huang, S.C., C.C. Tsai, and C.S. Sheu. 2000. Genetic analysis of chrysanthemum hybrids based on RAPD molecular markers. Bot. Bull. Acad. Sin. 41:257-262.

Johansson, B.G. 1972. Agarose gel electrophoresis. Scand. J. Clin. Lab. Invest. 29:7-19.

Kalia, R.K., M.K. Rai, S. Kalia, R. Singh, and A.K. Dhawan. 2011. Microsatellite markers: An overview of the recent progress in plants. Euphytica 177:309-334.

Kang, E., Y.M. Lee, S.Y. Sung, B.K. Ha, S.H. Kim, D.S. Kim, J.B. Kim, and S.Y. Kang. 2013. Analysis of the genetic relationship of gammairradiated in vitro mutants derived from standard-type chrysanthemum cv. Migok. Hort. Environ. Biotechnol. 54:76-81.

Koelling, J., M.C. Coles, P.D. Matthews, and A. Schwekendiek. 2012. Development of new microsatellite markers (SSRs) for Humulus lupulus. Mol. Breed. 30:479-484.

Leiss, K.A., F. Maltese, Y.H. Choi, R. Verpoorte, and P.G. Klinkhamer. 2009. Identification of chlorogenic acid as a resistance factor for thrips in chrysanthemum. Plant Physiol. 15:1567-1575. 
Lema-Rumiñska, J., M. Zalewska, and Z. Sadoch. 2004. Radiomutants of chrysanthemum (Dendranthema grandiflora Tzvelev) of the Lady group: RAPD analysis of the genetic diversity. Plant Breed. 123: 290-293.

Leutenegger, A.L., B. Prum, E. Génin, C. Verny, A. Lemainque, F. Clerget-Darpoux, and E.A. Thompson. 2003. Estimation of the inbreeding coefficient through use of genomic data. Amer. J. Hum. Genet. 73:516-523.

Li, Y., C. Huang, and R. Dong. 2012. Establishment and optimization of chrysanthemum SSR-PCR reaction system. Northern Hort. 13:127-131 [abstr.].

Liang, J., L. Zhao, R. Challis, and O. Leyser. 2010. Strigolactone regulation of shoot branching in chrysanthemum (Dendranthema grandiflorum). J. Expt. Bot. 61:3069-3078.

Lin, L.Z. and J.M. Harnly. 2010. Identification of the phenolic components of chrysanthemum flower (Chrysanthemum morifolium Ramat.). Food Chem. 120:319-326.

Lindqvist, C., A.C. Scheen, M.J. Yoo, P. Grey, D.G. Oppenheimer, J.H. Leebens-Mack, D.E. Soltis, P.S. Soltis, and V.A. Albert. 2006. An expressed sequence tag (EST) library from developing fruits of an Hawaiian endemic mint (Stenogyne rugosa, Lamiaceae): Characterization and microsatellite markers. BMC Plant Biol. 6:1471-2229.

Martí, C., E. Uberhuaga, and C. Pérez. 2002. Application of RAPD markers in the characterisation of chrysanthemum varieties and the assessment of somaclonal variation. Euphytica 127:247-253.

Ministry of Agriculture of the People's Republic of China. 2002. The handbook of distinctiveness, uniformity and stability test of new plant varieties of the People's Republic of China (chrysanthemum). Ministry of Agriculture of the People's Republic of China, Beijing, China.

Mori, G.M., M.I. Zucchi, I. Sampaio, and A.P. Souza. 2010. Microsatellites for the mangrove tree Avicennia germinans (Acanthaceae): Tools for hybridization and mating system studies. Amer. J. Bot. 97:e79-e81.

Nei, M. 1978. Estimation of average heterozygosity and genetic distance from a small number of individuals. Genetics 89:583-590.

Roxas, N.J.L., Y. Tashiro, S. Miyazaki, A. Takeshita, and T. Oshima. 1993. Isozyme analysis in higo chrysanthemum (Dendranthema grandiflora Tzvelev). J. Jpn. Soc. Hort. Sci. 61:919-924.
Sehrawat, S.K., R. Kumar, D.S. Dahiya, K.S. Boora, and R. Yadav. 2003. DNA fingerprinting of chrysanthemum cultivars using RAPDs. Acta Hort. 624:479-485.

Shao, Q., Q. Guo, Y. Deng, and H. Guo. 2010. A comparative analysis of genetic diversity in medicinal Chrysanthemum morifolium based on morphology, ISSR and SRAP markers. Biochem. Syst. Ecol. 38: 1160-1169.

Shapiro, A.L., E. Viñuela, and J.V. Maizel, Jr. 1967. Molecular weight estimation of polypeptide chains by electrophoresis in SDSpolyacrylamide gels. Biochem. Biophys. Res. Commun. 28:815.

Sheu, D.S., Y.T. Wang, and C.Y. Lee. 2000. Rapid detection of polyhydroxyalkanoate-accumulating bacteria isolated from the environment by colony PCR. Microbiology 146:2019-2025.

Squirrell, J., P.M. Hollingsworth, M. Woodhead, J. Russell, J. Lowe, M. Gibby, and W. Powell. 2003. How much effort is required to isolate nuclear microsatellites from plants? Mol. Ecol. 12:1339-1348.

Wang, H., J. Jiang, S. Chen, X. Qi, H. Peng, P. Li, A. Song, Z. Guan, W. Fang, Y. Liao, and F. Chen. 2013. Next-generation sequencing of the Chrysanthemum nankingense (Asteraceae) transcriptome permits large-scale unigene assembly and SSR marker discovery. PLoS One 8:e62293.

Wang, H., B. Zhang, Z. Wang, Y. Cheng, and Y. Ye. 2010a. Development and characterization of microsatellite loci in Taihangia rupestris (Rosaceae), a rare cliff herb. Amer. J. Bot. 97:e136-e138.

Wang, Z., H. Sun, H. Wang, and S. Ge. 2010b. Isolation and characterization of 50 nuclear microsatellite markers for Cathaya argyrophylla, a Chinese endemic conifer. Amer. J. Bot. 97:e117e120.

Wolff, K., E. Zietkiewicz, and H. Hofstra. 1995. Identification of chrysanthemum cultivars and stability of DNA fingerprint patterns. Theor. Appl. Genet. 91:439-447.

Zane, L., L. Bargelloni, and T. Patarnello. 2002. Strategies for microsatellite isolation: A review. Mol. Ecol. 11:1-16.

Zhang, F., S. Chen, F. Chen, W. Fang, Y. Chen, and F. Li. 2011. SRAPbased mapping and QTL detection for inflorescence-related traits in chrysanthemum (Chrysanthemum morifolium). Mol. Breed. 27:11-23.

Zhang, Y., M. Zhu, and S. Dai. 2013. Analysis of karyotype diversity of 40 Chinese chrysanthemum cultivars. J. Systematics Evol. 51: $335-352$. 